\title{
Análisis térmico de mieles de Trigona (Tetragonisca) angustula de Norte de Santander, Colombia
}

\author{
Thermal analysis of Trigona (Tetragonisca) angustula honeys from Norte de \\ Santander, Colombia
}

\section{Análise térmico de mel de Trigona (Tetragonisca) angustula de Norte de Santander, Colômbia}

\author{
Yaneth Cardona-Rodríguez ${ }^{1}$, Diana Alexandra Torres-Sánchez², Wolfgang Hoffmann ${ }^{3}$
}

Forma de citar: Y. Cardona-Rodríguez, D. A. Torres-Sánchez, W. Hoffmann. "Análisis térmico de mieles de Trigona (Tetragonisca) angustula de Norte de Santander, Colombia”, Respuestas, vol. 20, no. 2, pp. 135-144, 2015.

Recibido:

Enero 30 de 2015

Aceptado:

Mayo 29 de 2015
${ }^{1}$ Maestría en Química yaneth.cardona@unipamplona. edu.co

ORCID: 0000-0003-3323-7242

Universidad de Pamplona

Pamplona-Colombia

${ }^{2}$ Doctorado en Ciencias-Química datorres@unipamplona.edu.co ORCID: 0000-0002-1733-2904

Universidad de Pamplona Pamplona-Colombia

${ }^{3}$ Zootecnista vespula@gmx.de ORCID: 0000-0002-6359-8797

Universidad de Pamplona Pamplona-Colombia

\section{Resumen}

Antecedentes: Las mieles de abejas sin aguijón se utilizan principalmente con fines terapéuticos y medicinales; sin embargo, los estudios relacionados con estas mieles, son escasos y están enfocados en la determinación de algunas de sus características fisicoquímicas y microbianas. Objetivo: En este trabajo, se estudió el comportamiento térmico de mieles de Trigona (Tetragonisca) angustula, provenientes de tres lugares de Norte de Santander (Durania, Granja experimental Villa Marina y Los Patios), mediante calorimetría diferencial de barrido (DSC). Métodos: Se determinó el comportamiento térmico de las muestras de miel ( $\sim 10 \mathrm{mg})$, utilizando un equipo SDT-Q600 de TA Instruments, que realiza simultáneamente Análisis Termogravimétrico y Calorimetría Diferencial de Barrido (TGA/DSC). Resultados: Se encontró que las muestras presentan 4 transiciones térmicas, independientemente del sitio de muestreo. Adicionalmente, se encontraron diferencias estadísticamente significativas en las entalpías de dichas transiciones. Conclusión: Las mieles producidas por la especie T. angustula presentan un comportamiento térmico característico, que permite diferenciarlas según su procedencia geográfica.

Palabras Clave: Análisis térmico, Calorimetría Diferencial de Barrido (DSC), Miel, Trigona (Tetragonisca) angustula.
Julio - Diciembre 2015

ISSN 0122-820X

E-ISSN 2422-5053

PP: 135-144

\footnotetext{
Abstract

Background: Honey of stingless bees is used in traditional medicine; nevertheless, studies related to these honeys are scarce and are focused in physicochemical and microbiological properties. Objective: We studied thermal behavior of honeys produced by Trigona (Tetragonisca) angustula, collected from three different places (Durania, Villa Marina and Los Patios), using Differential Scanning Calorimetry (DSC). Methods: We determined thermal behavior of honey samples $(\sim 10 \mathrm{mg})$, using a SDT-Q600, TA Instruments, simultaneous differential scanning calorimetry/thermogravimetric analysis (DSC/TGA). Results: $T$. angustula honeys exhibited four thermal transitions regardless sampling place. In addition, we found significant differences in the enthalpies of these transitions.
} 
Conclusion: Honey produced by T. angustula exhibited a characteristic thermal behavior, that will allow differentiate its geographical origin.

Keywords: Thermal analysis, Differential Scanning Calorimetry (DSC), Honey, Trigona (Tetragonisca) angustula.

\section{Resumo}

Antecedentes: $\mathrm{O}$ mel de abelhas sem ferrão é usado principalmente com fins terapêuticos e medicinais; no entanto, os estudos relacionados com este tipo de mel, são escassos e estão focados na determinação de algumas de suas características físico-químicas e microbianas. Objetivo: Neste trabalho, se estudou o comportamento térmico do mel de Trigona (Tetragonisca) angustula, a partir de três lugares de Norte de Santander (Durania, Fazenda Experimental Villa Marina e Los Patios), através de calorimetria diferencial de varrimento (DSC). Metodologia: Determinou-se o comportamento térmico das amostras de mel $(\sim 10 \mathrm{mg})$, utilizando um equipo SDT-Q600 de TA Instruments, que realiza simultaneamente Análise Termogravimétrico e Calorimetria Diferencial de Varrimento (TGA/DSC). Resultados: Encontrou-se que as amostras apresentam quatro transições térmicas, independentemente do local de amostragem. Além disso, se encontraram diferenças estatisticamente significativas nas entalpias de tais transições. Conclusão: $\mathrm{O}$ mel produzido pela espécie T. angustula apresenta um comportamento térmico característico, que permite diferencia-a segundo sua procedência geográfica.

Palavras-chave: Análise térmico, Calorimetria Diferencial de Varrimento (DSC), Mel, Trigona (Tetragonisca) angustula.

\section{Introducción}

La supervivencia del ser humano en el planeta está supeditada a la existencia de otras especies animales y vegetales. La gran mayoría de los alimentos que consumimos está disponible gracias a la polinización y aproximadamente la mitad de los insectos que polinizan las plantas tropicales son abejas. En el Neotrópico se encuentran aproximadamente 6.000 especies de abejas, que con sus visitas a las flores en busca de néctar y polen se convierten en polinizadores eficientes [1]-[6].
Dentro de las diferentes tribus de abejas, se encuentra la tribu Meliponini que agrupa todas aquellas abejas conocidas como "abejas sin aguijón" encontradas en las áreas tropicales y subtropicales del mundo, que se caracterizan principalmente por tener aguijón reducido. Existen más de 500 especies en Colombia, agrupadas en 13 géneros y 10 subgéneros. Dentro de estos géneros, se encuentra el género Trigona, que está representado por abejas con alas más largas que su cuerpo y se encuentran en el Neotrópico desde México hasta Argentina y en la región Indoaustraliana. La especie más conocida de este género, es Trigona (Tetragonica) angustula, conocida comúnmente en Colombia como "abeja angelita". Los nidos de estas abejas constan de celdas de cría horizontales ubicadas en el centro del nido, protegidas por varias capas de cerumen (mezcla de cera secretada por las glándulas ceríferas mezclada con resinas de plantas); un orificio de entrada y las celdas (potes) donde se almacena el alimento (polen y miel) que se encuentran rodeando las celdas de cría (figura 1) [2], [7], [8].

Las abejas además de su importancia como polinizadores, son productoras de diversos tipos de mieles que dependiendo de la especie y su ubicación geográfica poseen diferentes propiedades nutritivas y terapéuticas. La miel comercializada mundialmente en su mayoría, corresponde a la producida por la especie Apis mellifera. En Colombia, específicamente en 
Norte de Santander, la producción en colmenas Langstroth es de aproximadamente $30 \mathrm{~kg} /$ colmena/año [9]. Por otra parte, las abejas pertenecientes a la tribu Meliponini producen diferentes cantidades de miel, conocida como "miel de pote" debido a que almacenan dicho alimento en un recipiente con forma de "pote u olla de barro" a diferencia de las celdas hexagonales utilizadas por la especie Apis mellifera. La cantidad de miel producida por los meliponinos oscila entre $0,5-2,5 \mathrm{~kg}$ por año. La miel de abejas sin aguijón es muy apreciada en los países americanos por su calidad y su consumo se relaciona con fines medicinales y terapéuticos más que como alimento, siendo su costo elevado, comparado con el precio de la miel común, producida por la especie Apis mellifera [10], [11].

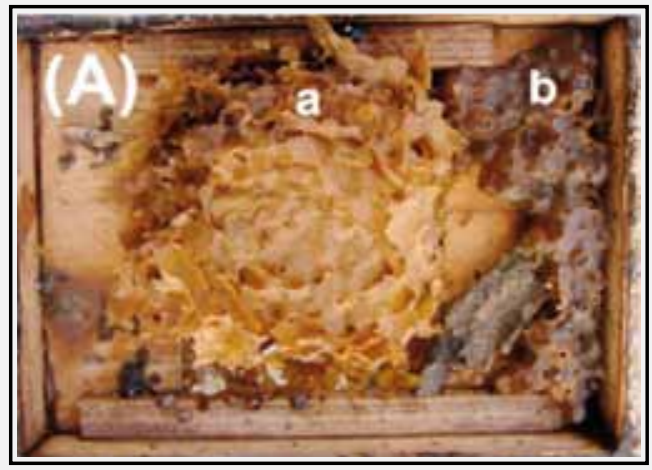

Figura 1. T. angustula con potes de miel en sus nidos. (A) Vista superior del nido, a. Celdas de cría, b. Potes de alimento y (B) Potes de miel.

Fuente: Grupo de Investigación Biocalorimetría, Universidad de Pamplona. del suelo, la especie de abejas y el estado fisiológico de la colonia [12], [13].

La caracterización de las mieles inició como respuesta a las demandas de los consumidores, en parte, debido a la aparición de adulteraciones. Por ello, la determinación de sus propiedades se utiliza para verificar la autenticidad del producto y revelar la posible presencia de componentes artificiales $\mathrm{o}$ adulterantes, lo que es muy importante para su comercio mundial; puesto que la miel se utiliza en una variedad cada vez mayor de productos alimenticios, medicinales y cosméticos. En los últimos años la calorimetría diferencial de barrido o DSC, acrónimo del nombre en inglés "Differential Scanning Calorimetry",
La miel está compuesta por aproximadamente 200 sustancias y tanto la producida por Apis mellifera como por otras especies, es una matriz poco favorable para el crecimiento de microorganismos, dado su alto contenido de azúcares, su carácter ácido y humedad relativamente baja. Los componentes minoritarios de la miel (en general) son compuestos nitrogenados, minerales, vitaminas (ácido ascórbico, niacina, piridoxina, etc.), ácidos orgánicos, proteínas, aminoácidos, enzimas (invertasa, glucosa oxidasa, catalasa, y fosfatasas), polen, compuestos fenólicos (ácidos fenólicos y flavonoides) y otros compuestos orgánicos. Estos componentes y la cantidad de los mismos, dependen de diversos factores como: las especies florales utilizadas, la naturaleza

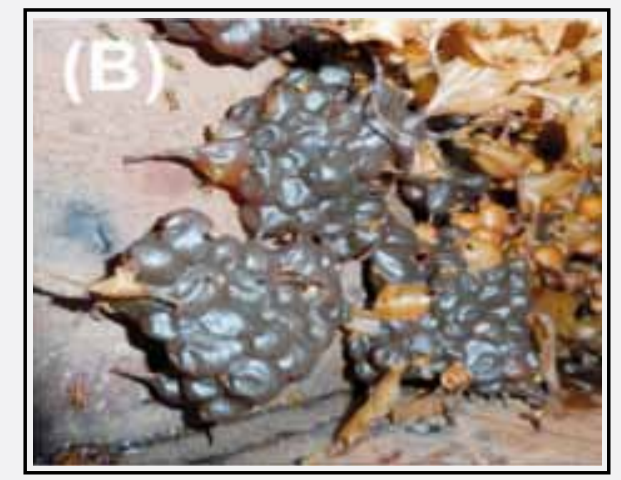

se ha utilizado ampliamente para establecer cambios físicos y químicos de diferentes muestras al aumentar su temperatura. Una de sus aplicaciones, es la detección de adulteraciones presentes en diferentes alimentos, incluidas las mieles. Esta técnica calorimétrica mide las diferencias en la cantidad de calor aportado a una sustancia y a un material de referencia en función de la temperatura de la muestra cuando las dos están sometidas a un programa controlado de temperatura, permitiendo determinar parámetros termodinámicos asociados a la transición inducida por el cambio de la temperatura en un periodo muy corto de tiempo, en comparación con otras técnicas [14]-[16]. 
No. 2

Julio - Diciembre 2015 ISSN 0122-820X E-ISSN 2422-5053 PP: $135-144$
En la actualidad, no se han reportado estudios térmicos de mieles de abejas sin aguijón, tal vez por el desconocimiento de las ventajas que ofrecen estas técnicas para determinar en corto tiempo la autenticidad de las mieles. Este estudio, muestra por primera vez el comportamiento térmico de mieles de la especie $T$. angustula proveniente de tres sitios diferentes, con la finalidad de establecer si las temperaturas y entalpías de las transiciones presentan diferencias significativas según la ubicación geográfica del nido.

\section{Materiales y métodos}

\subsection{Muestras}

Las muestras de miel (una por cada municipio) se recolectaron en epoca de cosecha (potes sellados) de colmenas racionales ubicadas en el casco urbano de Los patios y de Durania, y la Granja experimental Villa Marina, (km 49, vía Pamplona-Cúcuta, Vereda Matajira) en noviembre 2014, febrero 2015, y mayo de 2015, respectivamente. Se almacenaron en viales de vidrio color ambar a temperatura ambiente (aproximadamente $20^{\circ} \mathrm{C}$ ) y protegidas de la luz. Previamente a su análisis se homogenizaron mediante agitación magnética a una velocidad de 120 rpm durante 20 minutos [14]. Los sitios de muestreo fueron escogidos, teniendo en cuenta la ubicación geográfica de las especies elegidas como objeto del estudio.

\subsection{Equipos empleados}

Para la homogeneización de las muestras, se utilizó un agitador magnético Boeco. El estudio del comportamiento térmico se llevó a cabo mediante el uso del equipo SDT-Q600 de TA Instruments, que realiza simultáneamente TGA/DSC (análisis termogravimétrico/ análisis diferencial de barrido). El equipo se calibró usando zinc de alta pureza y zafiro como estandares de temperatura y flujo de calor respectivamente.

\subsection{Métodos}

Las muestras de miel ( $\sim 10 \mathrm{mg})$ se dispusieron en una celda de alúmina, y se analizaron por triplicado. El intervalo de temperatura utilizado fue desde temperatura ambiente $\left(\sim 20{ }^{\circ} \mathrm{C}\right)$ hasta $400{ }^{\circ} \mathrm{C}$, con una rampa de calentamiento de $10^{\circ} \mathrm{C} / \mathrm{min}$, usando nitrógeno $(100 \mathrm{~mL} / \mathrm{min})$ como gas de purga.

\subsection{Análisis estadístico}

Los valores de temperatura y entalpía, se determinaron utilizando el software TA Universal Analysis 2000 (TA instruments, Delaware, USA). Un análisis de varianza (ANOVA) $\quad(\alpha=0,05) \quad$ (utilizando como variable dependiente los valores de entalpìa de las diferentes transiciones y como variable independiente el lugar de procedencia de las muestras) y pruebas de múltiples rangos (Bonferroni), fueron realizados empleando el paquete estadístico Statgraphics Centurion (versión de prueba), con la finalidad de encontrar la existencia de diferencias estadísticamente significativas y conocer entre que grupos se dan dichas diferencias. utilizando análisis de un factor.

\section{Resultados y análisis}

Las muestras de miel exhibieron cuatro transiciones térmicas $\left(\operatorname{trs}_{1}, \operatorname{trs}_{2}, \operatorname{trs}_{3}, \operatorname{trs}_{4 \mathrm{a}}\right.$, $\operatorname{trs}_{4 \mathrm{~b}}$ ) (figura 2), independientemente del sitio de muestreo. En la tabla I se presentan los diferentes valores de temperatura $\left(\mathrm{T}_{\text {inicial }}\right.$, $\mathrm{T}_{\text {final }} \mathrm{y}_{\text {pico }}$ ) y entalpías para las muestras analizadas.

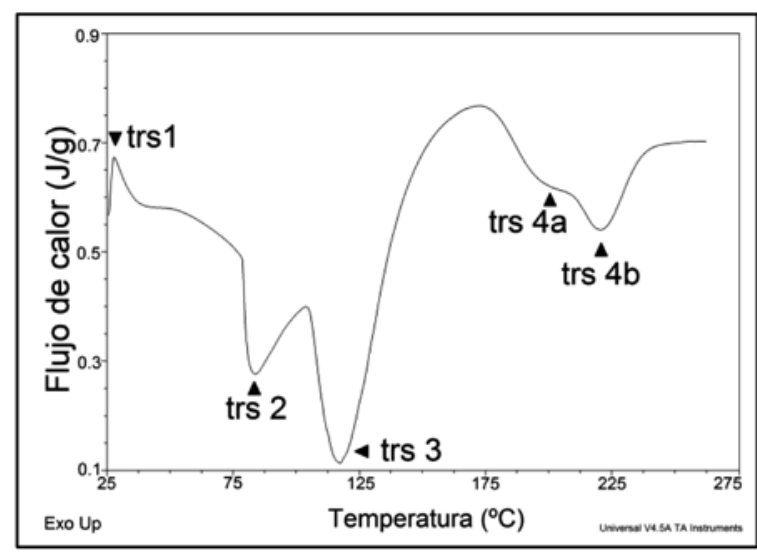

Figura 2. Termograma de miel producida por Trigona (tetragonisca) angustula, colectada en Los Patios (trs: transición). Fuente: Grupo de Investigación Biocalorimetría, Universidad de Pamplona. 

correspondientes.

\begin{tabular}{|c|c|c|c|c|}
\hline \multicolumn{2}{|c|}{ Transiciones } & Durania & Villa Marina & Los Patios \\
\hline \multirow[t]{4}{*}{1} & $\mathbf{T}_{\text {inicial }}{ }^{\circ} \mathbf{C}$ & $29,47 \pm 1,24$ & $30,33 \pm 0,22$ & $25,93 \pm 0,06$ \\
\hline & $\mathbf{T}_{\text {pico }}{ }^{\circ} \mathrm{C}$ & $32,89 \pm 0,23$ & $32,41 \pm 0,37$ & $28,48 \pm 0,06$ \\
\hline & $\mathbf{T}_{\text {final }}{ }^{\circ} \mathrm{C}$ & $42,63 \pm 1,26$ & $41,28 \pm 3,57$ & $44,00 \pm 0,89$ \\
\hline & Entalpía J/g & $17,69 \pm 0,82^{b}$ & $14,41 \pm 1,86^{a}$ & $13,84 \pm 0,78^{a}$ \\
\hline \multirow[t]{4}{*}{2} & $\mathrm{~T}_{\text {inicial }}{ }^{\circ} \mathrm{C}$ & $69,42 \pm 1,57$ & $54,89 \pm 3,54$ & $73,60 \pm 0,61$ \\
\hline & $\mathrm{T}_{\text {pice }}{ }^{\circ} \mathrm{C}$ & $80,70 \pm 3,61$ & $78,47 \pm 3,06$ & $82,29 \pm 0,52$ \\
\hline & $\mathbf{T}_{\text {final }}{ }^{\circ} \mathrm{C}$ & $108,85 \pm 1,58$ & $105,70 \pm 7,39$ & $100,47 \pm 0,43$ \\
\hline & Entalpía $\mathbf{J} / \mathbf{g}$ & $16,01 \pm 0,33^{b}$ & $28,18 \pm 4,26^{a}$ & $32,17 \pm 0,76^{a}$ \\
\hline \multirow[t]{4}{*}{3} & $\mathrm{~T}_{\text {inicial }}{ }^{\circ} \mathrm{C}$ & $110,51 \pm 1,16$ & $107,43 \pm 8,31$ & $101,92 \pm 0,85$ \\
\hline & $\mathbf{T}_{\text {pico }}{ }^{\circ} \mathrm{C}$ & $121,71 \pm 2,53$ & $113,86 \pm 6,91$ & $117,04 \pm 6,69$ \\
\hline & $\mathbf{T}_{\text {final }}{ }^{\circ} \mathbf{C}$ & $146,99 \pm 0,43$ & $132,56 \pm 0,71$ & $141,09 \pm 8,27$ \\
\hline & Entalpía $\mathbf{J} / \mathbf{g}$ & $55,71 \pm 4,08^{b}$ & $43,37 \pm 2,46^{a}$ & $46,62 \pm 3,89^{a}$ \\
\hline \multirow[t]{4}{*}{$4 a$} & $\mathbf{T}_{\text {inicial }}{ }^{\circ} \mathbf{C}$ & $175,88 \pm 1,22$ & $182,01 \pm 5,49$ & $173,80 \pm 4,58$ \\
\hline & $\mathbf{T}_{\text {pico }}{ }^{\circ} \mathrm{C}$ & $183,63 \pm 0,26$ & $186,36 \pm 0,34$ & $183,18 \pm 2,44$ \\
\hline & $\mathbf{T}_{\text {final }}{ }^{\circ} \mathbf{C}$ & $195,60 \pm 1,50$ & $195,75 \pm 0,18$ & $195,09 \pm 0,94$ \\
\hline & Entalpía $\mathbf{J} / \mathbf{g}$ & $9,81 \pm 0,63^{\mathrm{a}}$ & $9,50 \pm 1,73^{a}$ & $4,93 \pm 0,05^{b}$ \\
\hline \multirow[t]{4}{*}{$4 b$} & $\mathrm{~T}_{\text {inicial }}{ }^{\circ} \mathrm{C}$ & $205,60 \pm 0,15$ & $205,75 \pm 0,18$ & $197,38 \pm 1,06$ \\
\hline & $\mathrm{T}_{\text {pico }}{ }^{\circ} \mathrm{C}$ & $215,93 \pm 0,27$ & $215,15 \pm 2,35$ & $213,03 \pm 2,40$ \\
\hline & $\mathbf{T}_{\text {final }}{ }^{\circ} \mathbf{C}$ & $231,57 \pm 0,61$ & $230,19 \pm 0,18$ & $230,35 \pm 0,27$ \\
\hline & Entalpía J/g & $15,43 \pm 0,21^{a}$ & $15,84 \pm 0,40^{a}$ & $15,38 \pm 1,07^{a}$ \\
\hline
\end{tabular}

ISSN 0122-820X

Fuente: Grupo de Investigación Biocalorimetría, Universidad de Pamplona.

Las diferentes transiciones se presentaron en los siguientes rangos de temperatura: $25,92^{\circ} \mathrm{C}-44,96$ ${ }^{\circ} \mathrm{C}\left(\operatorname{trs}_{1}\right), 51,46^{\circ} \mathrm{C}-111,9^{\circ} \mathrm{C}\left(\operatorname{trs}_{2}\right), 98,1^{\circ} \mathrm{C}-149,56^{\circ} \mathrm{C}\left(\operatorname{trs}_{3}\right), 170,3{ }^{\circ} \mathrm{C}-196,11^{\circ} \mathrm{C}\left(\operatorname{trs}_{4 \mathrm{a}}\right)$ y 196,18 ${ }^{\circ} \mathrm{C}-232,05^{\circ} \mathrm{C}\left(\operatorname{trs}_{4 \mathrm{~b}}\right)$, con una entalpía promedio de: $15,31 \pm 2,10 \mathrm{~J} / \mathrm{g}, 25,45 \pm 7,61 \mathrm{~J} / \mathrm{g}, 48,57 \pm$ $6,34 \mathrm{~J} / \mathrm{g}, 8,08 \pm 2,54 \mathrm{~J} / \mathrm{g}$ y $15,55 \pm 0,62 \mathrm{~J} / \mathrm{g}$ respectivamente. Las entalpías y las temperaturas de cada transición de todas las mieles recolectadas en los diferentes sitios de muestreo, se presentan en la tabla I y en las figuras 3 y 4 . La mayor variación de entalpía, se presentó en la transición 2 para la miel proveniente de Villa Marina $(\boldsymbol{\sigma}=4,26 ; \mathrm{CV}=15,11 \%)$ y la menor, en la transición 4a para la miel originaria de Los Patios $(\boldsymbol{\sigma}=0,05 ; \mathrm{CV}=0,93 \%)$.

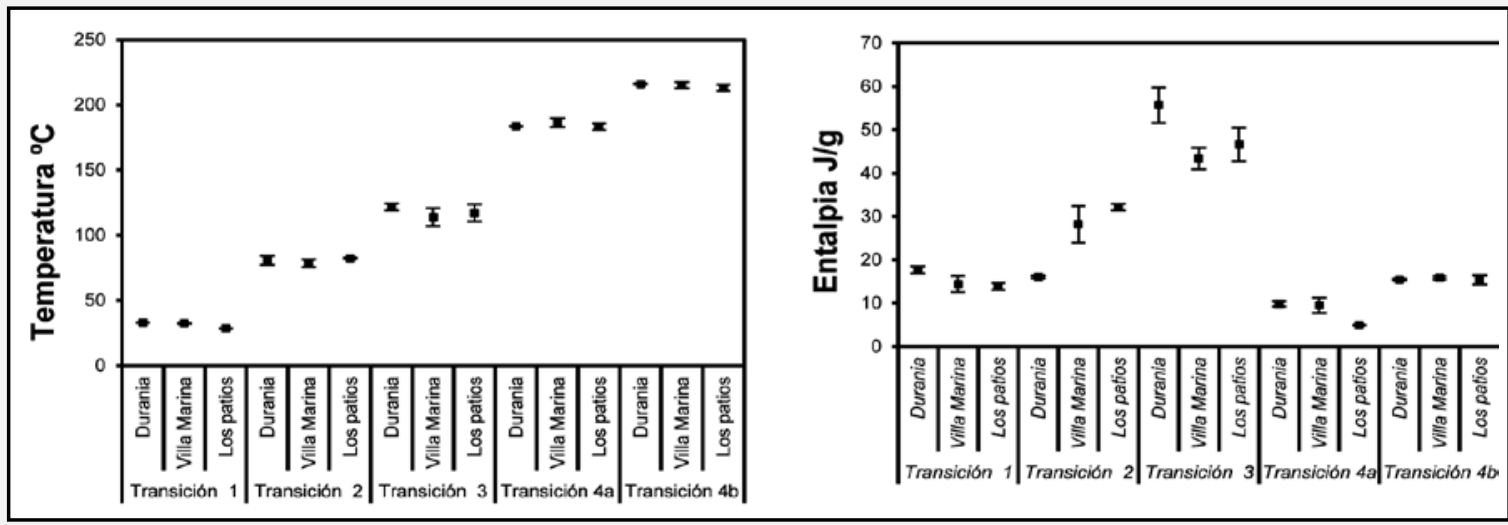

Figura 3. Temperatura y entalpía de las transiciones de las muestras de miel. Fuente: Grupo de Investigación Biocalorimetría, Universidad de Pamplona. 
No. 2

Julio - Diciembre 2015 ISSN 0122-820X E-ISSN 2422-5053 PP: 135-144
Para establecer que cambios físicos y químicos se dan en las diferentes transiciones térmicas observadas en la miel a medida que se aumenta su temperatura, se requiere tener en cuenta lo reportado en diferentes estudios con respecto a los valores de las propiedades fisicoquímicas para las mieles T. angustula de diferentes países; así, por ejemplo, la literatura reporta un porcentaje de humedad de 20,5\% y $24,3 \pm 2,3 \%$ (Colombia) [8], [12]; 24,4 \pm $0,8 \%$ (Brasil) [17]; 17,5 $\pm 2,8 \%$ (Guatemala)
[18] y 22,4 $\pm 1,3 \%$ (Venezuela) [19]. Por otra parte, el contenido de azúcares y proteínas también ha sido reportado para mieles de esta especie, mostrando un contenido de azúcares reductores de 65,9 - 69,4\% [8], [20] y de proteínas $1,19 \%$ [18]. Estos valores confirman la presencia de humedad, azúcares, proteínas y compuestos volátiles a los cuales se deben los valores observados en las entalpías de las transiciones.

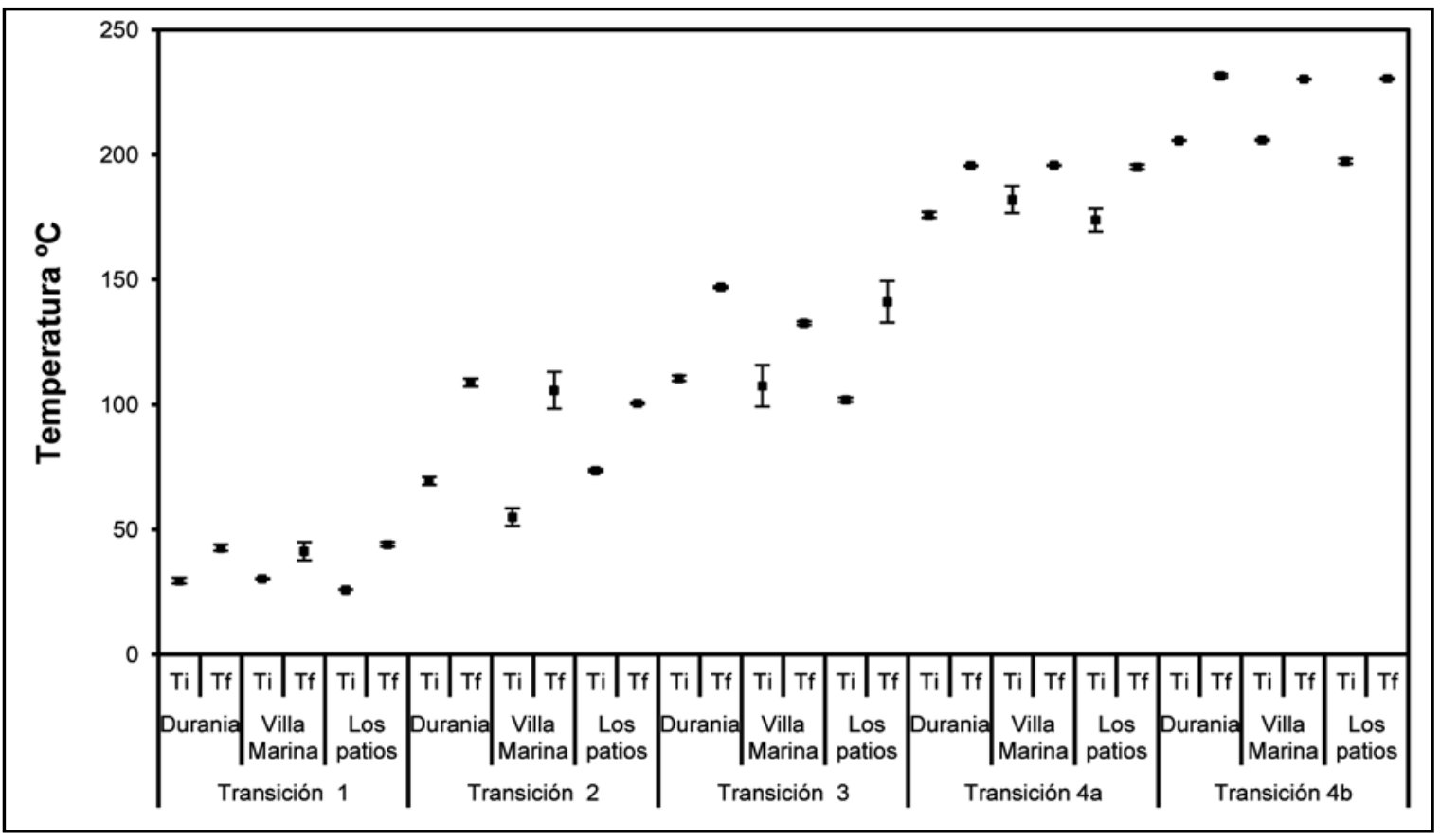

Figura 4. Temperaturas inicial $\left(\mathrm{T}_{\mathrm{i}}\right)$ y final $\left(\mathrm{T}_{\mathrm{f}}\right)$ para las transiciones $\left(\operatorname{trs}_{1}-\operatorname{trs}_{4 \mathrm{~b}}\right)$ presentes en la miel de T. angustula. Fuente: Grupo de Investigación Biocalorimetría, Universidad de Pamplona.

Teniendo en cuenta las temperaturas a las que se presentan las diferentes transiciones y la composición de la miel de T. angustula, especificada en el párrafo anterior, se podría decir que: la primera transición $\left(\operatorname{trs}_{1}\right)$ se relaciona con la vaporización de los compuestos volátiles, las transiciones 2 y $3\left(\operatorname{trs}_{2}, \operatorname{trs}_{3}\right)$ con evaporación de agua y desnaturalización de proteínas, además, del inicio de la fusión de algunos azúcares presentes en la muestra, y la cuarta transición $\left(\operatorname{trs}_{4 a}, \operatorname{trs}_{4 b}\right)$ con la degradación y fusión de los azúcares. Lo anterior puede explicarse teniendo en cuenta que: los compuestos volátiles suelen ser extraídos y determinados en el rango de temperatura en el cual se presenta la primera transición [21]-[26], las proteínas presentan fenómenos térmicos en el rango de las transiciones dos y tres [27][32] y la temperatura de descomposición de los azúcares corresponde a la temperatura de inicio de la fusión de los mismos, la cual se presenta en el rango de la cuarta transición $\left(\operatorname{trs}_{4 \mathrm{a}}, \operatorname{trs}_{4 \mathrm{~b}}\right)$ [33].

Debido a que la disponibilidad floral puede diferir respecto al lugar en el cual se encuentran las colmenas y a la época del año, se realizó un análisis estadístico ANOVA de un factor, el cual permitió determinar que el factor lugar 
efectivamente tiene efecto estadísticamente significativo $(p<0,05)$, sobre la variable entalpía de las transiciones $\operatorname{trs}_{1}-\operatorname{trs}_{4 \mathrm{~b}}$ y sobre las temperaturas de dichas transiciones $(\mathrm{p}<0,05)$, con un $95 \%$ de nivel de confianza.

Adicionalmente, mediante un análisis estadístico ANOVA simple, se encontró que no hay diferencias estadísticamente significativas entre las entalpías de las diferentes transiciones para las mieles proveniente de Los patios y Villa Marina ( $p>0,05)$, pero sí hay diferencias entre estas mieles con la de Durania $(p<0,05)$ en la entalpía de las transiciones $\operatorname{trs}_{1}, \operatorname{trs}_{2}, \operatorname{trs}_{3}$ $y \operatorname{trs}_{4 \mathrm{a}}$. La transición $\operatorname{trs}_{4 \mathrm{~b}}$, mostró que no existe diferencia estadísticamente significativa ( $p>0,05)$ entre las mieles colectadas en los diferentes lugares, con un nivel del $95 \%$ de confianza.

\section{Conclusiones}

Las muestras de miel provenientes de los diferentes sitios, a pesar de exhibir las mismas transiciones $\left(\operatorname{trs}_{1}, \operatorname{trs}_{2}, \operatorname{trs}_{3}, \operatorname{trs}_{4 \mathrm{a}}, \operatorname{trs}_{4 \mathrm{~b}}\right)$, presentan diferencias significativas en los valores de entalpía de las transiciones $\operatorname{trs}_{1}$, $\operatorname{trs}_{2}, \operatorname{trs}_{3}, \operatorname{trs}_{4 \mathrm{a}}$.

Las transiciones térmicas (entalpía y temperatura de aparición) pueden ser utilizadas junto con otros análisis como cromatografía de gases acoplada a espectrometría de masas (GC-MS), espectrometría de absorción atómica (AA), y análisis polínico; para establecer diferencias en cuanto al origen botánico de las mieles.

La calorimetría diferencial de barrido (DSC) podría utilizarse para determinar la procedencia (huella dactilar) de la miel de $T$. angustula.

\section{Agradecimientos}

Los autores agradecen a la Universidad de Pamplona, Vicerrectoría de Investigaciones (Programa de movilidad) por la financiación de la asistencia como ponente de Y. Cardona al CLAQ 2014, realizado en Lima, Perú.

\section{Referencias}

Julio - Diciembre 2015 ISSN 0122-820X

[1] H. M. Habib, F. T. Al Meqbali, H. Kamal, U. D. Souka and W. H. Ibrahim, "Physicochemical and biochemical properties of honeys from arid regions," Food Chemistry, vol. 153, pp. 35-43, June 2014.

[2] G. Nates-Parra y J. M. Rosso-Londoño, "Diversidad de abejas sin aguijón (Hymenoptera:Meliponini) utilizadas en meliponicultura en Colombia," Acta Biológica Colombiana, vol. 18, pp. 415-426, 2013.

[3] G. Nates-Parra, "Abejas silvestres y polinización," Manejo Integrado de Plagas y Agroecología, vol. 75, pp. 7-20, 2005.

[4] C. D. Michener, "The importance of Bees," in The Bees of the world, ed Baltimore: John Hopkins University Press, 2007, pp. 4-5.

[5] M. Juan-Borrás, E. Domenech, M. Hellebrandova and I. Escriche, "Effect of country origin on physicochemical, sugar and volatile composition of acacia, sunflower and tilia honeys," Food Research International, vol. 60, pp. 86-94, June 2014.

[6] I. K. Karabagias, A. Badeka, S. Kontakos, S. Karabournioti and M. G. Kontominas, "Characterisation and classification of Greek pine honeys according to their geographical origin based on volatiles, physicochemical parameters and chemometrics," Food Chemistry, vol. 146, pp. 548-557, March 2014.

[7] A. Torres, W. Hoffmann and I. Lamprecht, "Thermal investigations of a nest of the stingless bee Trigona (Frieseomelitta) nigra paupera provancher in Colombia," Journal of
E-ISSN 2422-5053

PP: $135-144$ 
No. 2

[8] A. Torres, A. Garedew, E. Schmolz and I. Lamprecht, "Calorimetric investigation of the antimicrobial action and insight into the chemical properties of "angelita" honey - a product of the stingless bee Tetragonisca angustula from Colombia," Thermochimica Acta, vol. 415, no. 1-2, pp. 107-113, June 2004.

[9] J. Laverde Rodríguez, L. M. Egea Hernández, D. M. Rodrígez Zárate y J. E. Peña Saenz, "Agenda prospectiva de investigación y desarrollo tecnológico para la cadena productiva de las abejas y la apicultura en colombia con énfasis en miel de abejas. ," M. d. A. y. D. Rural., Ed., ed. Bogotá - Colombia: Ministerio de Agricultura y Desarrollo Rural., 2010, p. 224.

[10] M. Cortopassi-Laurino, V. L. Imperatriz-Fonseca, D. W. Roubik, A. Dollin, T. Heard, I. Aguilar, et al., "Global meliponiculture: challenges and opportunities," Apidologie, vol. 37, pp. 275-292, 2006.

[11] G. Nates-Parra, "Las Abejas sin Aguijón (Hymenoptera: Apidae: Meliponini) de Colombia," Biota Colombiana, vol. 2, pp. 233-248, 2001.
Meliponini): interspecific variation and comparison with honey of Apis mellifera," Apidologie, vol. 37, pp. 480486, 2006.

[14] C. Cordella, J. F. Antinelli, C. Aurieres, J. P. Faucon, D. Cabrol-Bass and N. Sbirrazzuoli, "Use of differential scanning calorimetry (DSC) as a new technique for detection of adulteration in honeys. 1. Study of adulteration effect on honey thermal behavior," Journal of Agricultural and Food Chemistry, vol. 50, no. 1, pp. 203-208, January 2002.

[15] C. Cordella, J. P. Faucon, D. CabrolBass, and N. Sbirrazzuoli, "Application of DSC as a tool for honey floral species characterization and adulteration detection," Journal of Thermal Analysis and Calorimetry, vol. 71, no. 1, pp. 279290, January 2003.

[16] O. Dahimi, A. A. Rahim, S. M. Abdulkarim, M. S. Hassan, S. B. T. Z. Hashari, A. Siti Mashitoh, et al., "Multivariate statistical analysis treatment of DSC thermal properties for animal fat adulteration," Food Chemistry, vol. 158, pp. 132-138, September 2014.

[17] L. B. de Almeida-Muradian, "Tetragonisca angustula Pot-Honey Compared to Apis mellifera Honey from Brazil," in Pot-Honey, P. Vit, S. R. M. Pedro, and D. Roubik, Eds., ed: Springer New York, 2013, pp. 375-382.

[18] M. J. Dardón, C. Maldonado-Aguilera, and E. Enríquez, "The Pot-Honey of Guatemalan Bees," in Pot Honey, P. Vit, S. R. M. Pedro, and D. Roubik, Eds., ed: Springer New York, 2013, pp. 395408.

[13] L. Bijlsma, L. L. M. De Bruijn, E. P. Martens and M. J. Sommeijer, "Water content of stingless bee honeys (Apidae,
[19] P. Vit, L. Oddo, Persano, M. Marano, Luisa and E. Salas de Mejias, 
"Venezuelan stingless bee honeys characterized by multivariate analysis of physicochemical properties," Apidologie, vol. 29, pp. 377-389, 1998.

[20] M. J. Dardón y E. Enríquez, "Caracterización fisicoquímica $\mathrm{y}$ antimicrobiana de la miel de nueve especies de abejas sin aguijón (Meliponini) de Guatemala," Interciencia, vol. 33, pp. 916-922, 2008.

[21] L. Piasenzotto, L. Gracco and L. Conte, "Solid phase microextraction (SPME) applied to honey quality control," Journal of the Science of Food and Agriculture, vol. 83, pp. 1037-1044, 2003.

[22] T. Wolski , K. Tambor, H. RybakChmielewska and B. Kêdzia, "Identification of honey volatile components by Solid Phase Microextraction (SPME) and Gas Chromatography /Mass Spectrometry (GC/MS)," Journal of Apicultural Science, vol. 50, pp. 115-126, 2006.

[23] R. A. Perez, C. Sanchez-Brunete, R. M. Calvo, and J. L. Tadeo, "Analysis of volatiles from Spanish honeys by Solid-Phase Microextraction and Gas Chromatography / Mass Spectrometry," Journal of Agricultural and Food Chemistry vol. 50, pp. 2633-7, April 2002.

[24] M. P. Gianelli Barra, M. C. Ponce-Díaz, and C. Venegas-Gallegos, "Volatile Compounds in Honey Produced in the Central Valley of Nuble Province, Chile," Chilean Journal of Agricultural Research, vol. 70, pp. 75-84, 2010.

[25] F. Pasini, S. Gardini, G. L. Marcazzan, and M. F. Caboni, "Buckwheat honeys: screening of composition and properties," Food Chemistry, vol.
141, pp. 2802-11, Dec 1 2013.[26]

F. Pasini, S. Gardini, G. L. Marcazzan,
and M. F. Caboni, "Buckwheat $0122-820 \mathrm{X}$
honeys: screening of composition and
properties," Food Chemistry, vol. 141,
pp. 2802-2811, December 2013.
M. Mizuno and M. J. Pikal, "Is the
pre-Tg DSC endotherm observed with
solid state proteins associated with the
protein internaldynamics? Investigation
of bovine serum albumin by solid
state hydrogen/deuterium exchange,"
European Journal of Pharmaceutics
and Biopharmaceutics, vol. 85, pp.
170-176, October 2013.
J. C. Martinez, A. R. Viguera, L. Serrano,
V. V. Filimonov, and P. L. Mateo, "The
DSC data analysis for small, single-
domain proteins. Application to the
SH3 domain," Reactive and Functional
Polymers, vol. 36, pp. 221-225, May
1998.

F. Pasini, S. Gardini, G. L. Marcazzan,
and M. F. Caboni, "Buckwheat $0122-820 \mathrm{X}$
honeys: screening of composition and
properties," Food Chemistry, vol. 141,
pp. 2802-2811, December 2013.
M. Mizuno and M. J. Pikal, "Is the
pre-Tg DSC endotherm observed with
solid state proteins associated with the
protein internaldynamics? Investigation
of bovine serum albumin by solid
state hydrogen/deuterium exchange,"
European Journal of Pharmaceutics
and Biopharmaceutics, vol. 85, pp.
170-176, October 2013.
J. C. Martinez, A. R. Viguera, L. Serrano,
V. V. Filimonov, and P. L. Mateo, "The
DSC data analysis for small, single-
domain proteins. Application to the
SH3 domain," Reactive and Functional
Polymers, vol. 36, pp. 221-225, May
1998.

F. Pasini, S. Gardini, G. L. Marcazzan,
and M. F. Caboni, "Buckwheat $0122-820 \mathrm{X}$
honeys: screening of composition and
properties," Food Chemistry, vol. 141,
pp. 2802-2811, December 2013.
M. Mizuno and M. J. Pikal, "Is the
pre-Tg DSC endotherm observed with
solid state proteins associated with the
protein internaldynamics? Investigation
of bovine serum albumin by solid
state hydrogen/deuterium exchange,"
European Journal of Pharmaceutics
and Biopharmaceutics, vol. 85, pp.
170-176, October 2013.
J. C. Martinez, A. R. Viguera, L. Serrano,
V. V. Filimonov, and P. L. Mateo, "The
DSC data analysis for small, single-
domain proteins. Application to the
SH3 domain," Reactive and Functional
Polymers, vol. 36, pp. 221-225, May
1998.

F. Pasini, S. Gardini, G. L. Marcazzan,
and M. F. Caboni, "Buckwheat $0122-820 \mathrm{X}$
honeys: screening of composition and
properties," Food Chemistry, vol. 141,
pp. 2802-2811, December 2013.
M. Mizuno and M. J. Pikal, "Is the
pre-Tg DSC endotherm observed with
solid state proteins associated with the
protein internaldynamics? Investigation
of bovine serum albumin by solid
state hydrogen/deuterium exchange,"
European Journal of Pharmaceutics
and Biopharmaceutics, vol. 85, pp.
170-176, October 2013.
J. C. Martinez, A. R. Viguera, L. Serrano,
V. V. Filimonov, and P. L. Mateo, "The
DSC data analysis for small, single-
domain proteins. Application to the
SH3 domain," Reactive and Functional
Polymers, vol. 36, pp. 221-225, May
1998.

F. Pasini, S. Gardini, G. L. Marcazzan,
and M. F. Caboni, "Buckwheat $0122-820 \mathrm{X}$
honeys: screening of composition and
properties," Food Chemistry, vol. 141,
pp. 2802-2811, December 2013.
M. Mizuno and M. J. Pikal, "Is the
pre-Tg DSC endotherm observed with
solid state proteins associated with the
protein internaldynamics? Investigation
of bovine serum albumin by solid
state hydrogen/deuterium exchange,"
European Journal of Pharmaceutics
and Biopharmaceutics, vol. 85, pp.
170-176, October 2013.
J. C. Martinez, A. R. Viguera, L. Serrano,
V. V. Filimonov, and P. L. Mateo, "The
DSC data analysis for small, single-
domain proteins. Application to the
SH3 domain," Reactive and Functional
Polymers, vol. 36, pp. 221-225, May
1998.

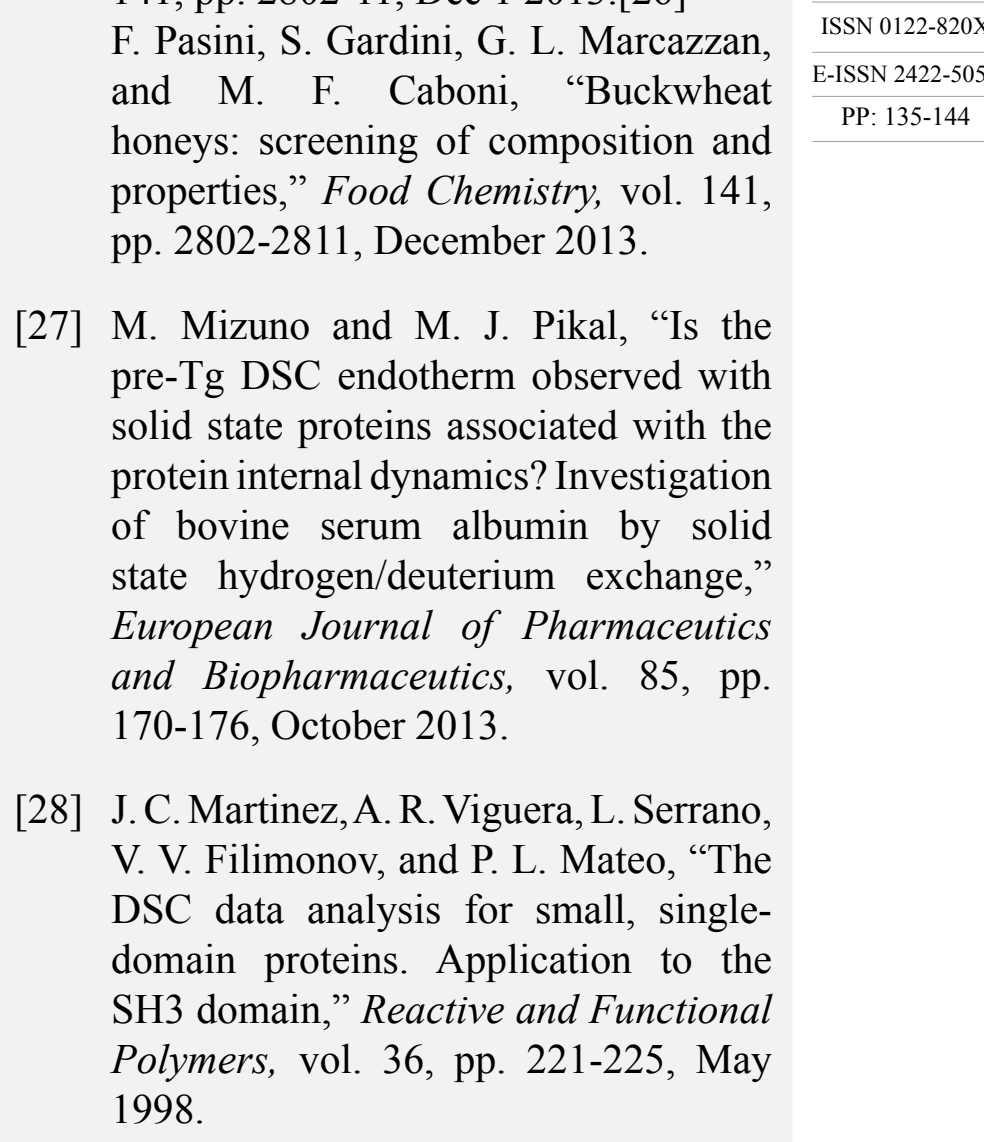

F. Pasini, S. Gardini, G. L. Marcazzan,
and M. F. Caboni, "Buckwheat $0122-820 \mathrm{X}$
honeys: screening of composition and
properties," Food Chemistry, vol. 141,
pp. 2802-2811, December 2013.
M. Mizuno and M. J. Pikal, "Is the
pre-Tg DSC endotherm observed with
solid state proteins associated with the
protein internaldynamics? Investigation
of bovine serum albumin by solid
state hydrogen/deuterium exchange,"
European Journal of Pharmaceutics
and Biopharmaceutics, vol. 85, pp.
170-176, October 2013.
J. C. Martinez, A. R. Viguera, L. Serrano,
V. V. Filimonov, and P. L. Mateo, "The
DSC data analysis for small, single-
domain proteins. Application to the
SH3 domain," Reactive and Functional
Polymers, vol. 36, pp. 221-225, May
1998.

F. Pasini, S. Gardini, G. L. Marcazzan,
and M. F. Caboni, "Buckwheat $0122-820 \mathrm{X}$
honeys: screening of composition and
properties," Food Chemistry, vol. 141,
pp. 2802-2811, December 2013.
M. Mizuno and M. J. Pikal, "Is the
pre-Tg DSC endotherm observed with
solid state proteins associated with the
protein internaldynamics? Investigation
of bovine serum albumin by solid
state hydrogen/deuterium exchange,"
European Journal of Pharmaceutics
and Biopharmaceutics, vol. 85, pp.
170-176, October 2013.
J. C. Martinez, A. R. Viguera, L. Serrano,
V. V. Filimonov, and P. L. Mateo, "The
DSC data analysis for small, single-
domain proteins. Application to the
SH3 domain," Reactive and Functional
Polymers, vol. 36, pp. 221-225, May
1998.

F. Pasini, S. Gardini, G. L. Marcazzan,
and M. F. Caboni, "Buckwheat $0122-820 \mathrm{X}$
honeys: screening of composition and
properties," Food Chemistry, vol. 141,
pp. 2802-2811, December 2013.
M. Mizuno and M. J. Pikal, "Is the
pre-Tg DSC endotherm observed with
solid state proteins associated with the
protein internaldynamics? Investigation
of bovine serum albumin by solid
state hydrogen/deuterium exchange,"
European Journal of Pharmaceutics
and Biopharmaceutics, vol. 85, pp.
170-176, October 2013.
J. C. Martinez, A. R. Viguera, L. Serrano,
V. V. Filimonov, and P. L. Mateo, "The
DSC data analysis for small, single-
domain proteins. Application to the
SH3 domain," Reactive and Functional
Polymers, vol. 36, pp. 221-225, May
1998.

F. Pasini, S. Gardini, G. L. Marcazzan,
and M. F. Caboni, "Buckwheat $0122-820 \mathrm{X}$
honeys: screening of composition and
properties," Food Chemistry, vol. 141,
pp. 2802-2811, December 2013.
M. Mizuno and M. J. Pikal, "Is the
pre-Tg DSC endotherm observed with
solid state proteins associated with the
protein internaldynamics? Investigation
of bovine serum albumin by solid
state hydrogen/deuterium exchange,"
European Journal of Pharmaceutics
and Biopharmaceutics, vol. 85, pp.
170-176, October 2013.
J. C. Martinez, A. R. Viguera, L. Serrano,
V. V. Filimonov, and P. L. Mateo, "The
DSC data analysis for small, single-
domain proteins. Application to the
SH3 domain," Reactive and Functional
Polymers, vol. 36, pp. 221-225, May
1998.

F. Pasini, S. Gardini, G. L. Marcazzan,
and M. F. Caboni, "Buckwheat $0122-820 \mathrm{X}$
honeys: screening of composition and
properties," Food Chemistry, vol. 141,
pp. 2802-2811, December 2013.
M. Mizuno and M. J. Pikal, "Is the
pre-Tg DSC endotherm observed with
solid state proteins associated with the
protein internaldynamics? Investigation
of bovine serum albumin by solid
state hydrogen/deuterium exchange,"
European Journal of Pharmaceutics
and Biopharmaceutics, vol. 85, pp.
170-176, October 2013.
J. C. Martinez, A. R. Viguera, L. Serrano,
V. V. Filimonov, and P. L. Mateo, "The
DSC data analysis for small, single-
domain proteins. Application to the
SH3 domain," Reactive and Functional
Polymers, vol. 36, pp. 221-225, May
1998.

F. Pasini, S. Gardini, G. L. Marcazzan,
and M. F. Caboni, "Buckwheat $0122-820 \mathrm{X}$
honeys: screening of composition and
properties," Food Chemistry, vol. 141,
pp. 2802-2811, December 2013.
M. Mizuno and M. J. Pikal, "Is the
pre-Tg DSC endotherm observed with
solid state proteins associated with the
protein internaldynamics? Investigation
of bovine serum albumin by solid
state hydrogen/deuterium exchange,"
European Journal of Pharmaceutics
and Biopharmaceutics, vol. 85, pp.
170-176, October 2013.
J. C. Martinez, A. R. Viguera, L. Serrano,
V. V. Filimonov, and P. L. Mateo, "The
DSC data analysis for small, single-
domain proteins. Application to the
SH3 domain," Reactive and Functional
Polymers, vol. 36, pp. 221-225, May
1998.

F. Pasini, S. Gardini, G. L. Marcazzan,
and M. F. Caboni, "Buckwheat $0122-820 \mathrm{X}$
honeys: screening of composition and
properties," Food Chemistry, vol. 141,
pp. 2802-2811, December 2013.
M. Mizuno and M. J. Pikal, "Is the
pre-Tg DSC endotherm observed with
solid state proteins associated with the
protein internaldynamics? Investigation
of bovine serum albumin by solid
state hydrogen/deuterium exchange,"
European Journal of Pharmaceutics
and Biopharmaceutics, vol. 85, pp.
170-176, October 2013.
J. C. Martinez, A. R. Viguera, L. Serrano,
V. V. Filimonov, and P. L. Mateo, "The
DSC data analysis for small, single-
domain proteins. Application to the
SH3 domain," Reactive and Functional
Polymers, vol. 36, pp. 221-225, May
1998.

F. Pasini, S. Gardini, G. L. Marcazzan,
and M. F. Caboni, "Buckwheat $0122-820 \mathrm{X}$
honeys: screening of composition and
properties," Food Chemistry, vol. 141,
pp. 2802-2811, December 2013.
M. Mizuno and M. J. Pikal, "Is the
pre-Tg DSC endotherm observed with
solid state proteins associated with the
protein internaldynamics? Investigation
of bovine serum albumin by solid
state hydrogen/deuterium exchange,"
European Journal of Pharmaceutics
and Biopharmaceutics, vol. 85, pp.
170-176, October 2013.
J. C. Martinez, A. R. Viguera, L. Serrano,
V. V. Filimonov, and P. L. Mateo, "The
DSC data analysis for small, single-
domain proteins. Application to the
SH3 domain," Reactive and Functional
Polymers, vol. 36, pp. 221-225, May
1998.

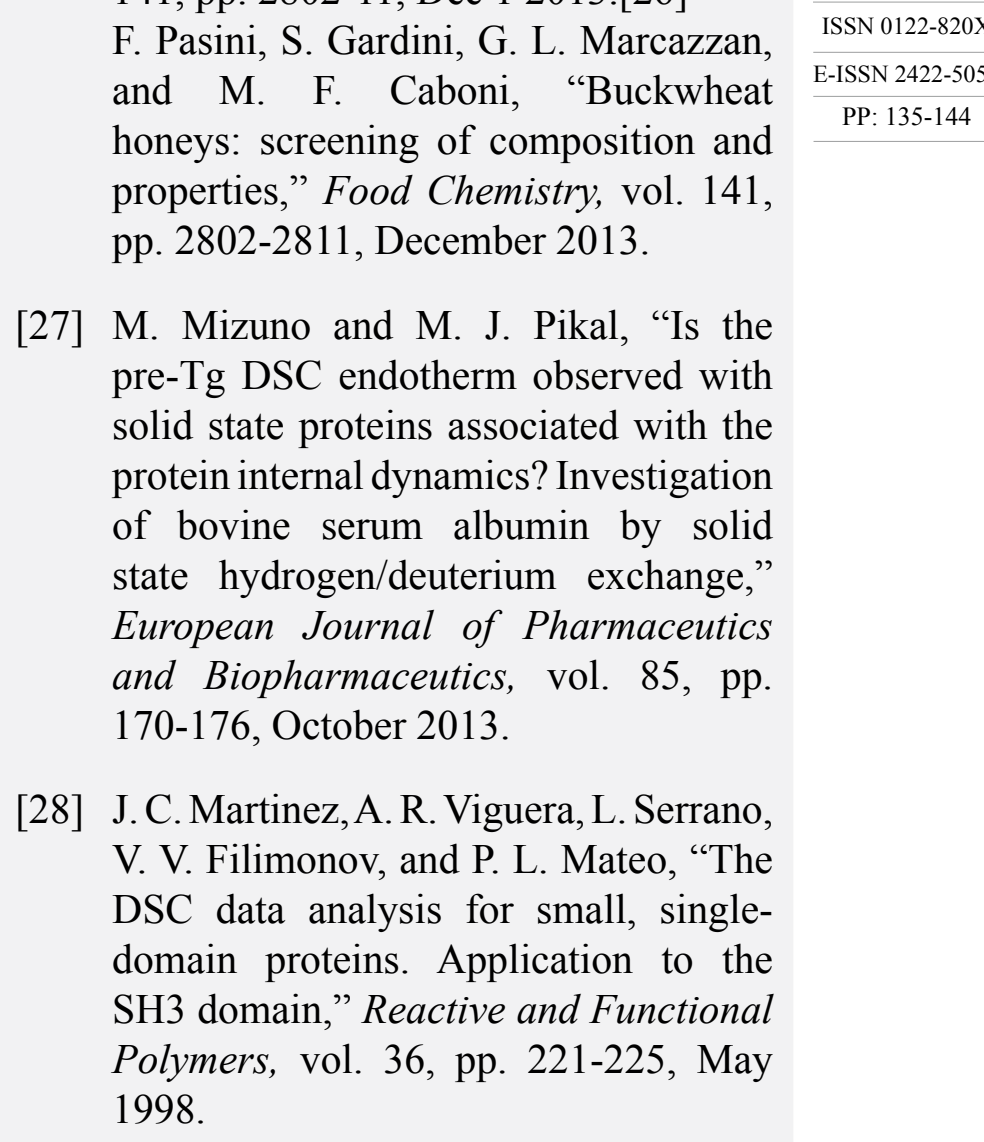

F. Pasini, S. Gardini, G. L. Marcazzan,
and M. F. Caboni, "Buckwheat $0122-820 \mathrm{X}$
honeys: screening of composition and
properties," Food Chemistry, vol. 141,
pp. 2802-2811, December 2013.
M. Mizuno and M. J. Pikal, "Is the
pre-Tg DSC endotherm observed with
solid state proteins associated with the
protein internaldynamics? Investigation
of bovine serum albumin by solid
state hydrogen/deuterium exchange,"
European Journal of Pharmaceutics
and Biopharmaceutics, vol. 85, pp.
170-176, October 2013.
J. C. Martinez, A. R. Viguera, L. Serrano,
V. V. Filimonov, and P. L. Mateo, "The
DSC data analysis for small, single-
domain proteins. Application to the
SH3 domain," Reactive and Functional
Polymers, vol. 36, pp. 221-225, May
1998.

F. Pasini, S. Gardini, G. L. Marcazzan,
and M. F. Caboni, "Buckwheat $0122-820 \mathrm{X}$
honeys: screening of composition and
properties," Food Chemistry, vol. 141,
pp. 2802-2811, December 2013.
M. Mizuno and M. J. Pikal, "Is the
pre-Tg DSC endotherm observed with
solid state proteins associated with the
protein internaldynamics? Investigation
of bovine serum albumin by solid
state hydrogen/deuterium exchange,"
European Journal of Pharmaceutics
and Biopharmaceutics, vol. 85, pp.
170-176, October 2013.
J. C. Martinez, A. R. Viguera, L. Serrano,
V. V. Filimonov, and P. L. Mateo, "The
DSC data analysis for small, single-
domain proteins. Application to the
SH3 domain," Reactive and Functional
Polymers, vol. 36, pp. 221-225, May
1998.

F. Pasini, S. Gardini, G. L. Marcazzan,
and M. F. Caboni, "Buckwheat $0122-820 \mathrm{X}$
honeys: screening of composition and
properties," Food Chemistry, vol. 141,
pp. 2802-2811, December 2013.
M. Mizuno and M. J. Pikal, "Is the
pre-Tg DSC endotherm observed with
solid state proteins associated with the
protein internaldynamics? Investigation
of bovine serum albumin by solid
state hydrogen/deuterium exchange,"
European Journal of Pharmaceutics
and Biopharmaceutics, vol. 85, pp.
170-176, October 2013.
J. C. Martinez, A. R. Viguera, L. Serrano,
V. V. Filimonov, and P. L. Mateo, "The
DSC data analysis for small, single-
domain proteins. Application to the
SH3 domain," Reactive and Functional
Polymers, vol. 36, pp. 221-225, May
1998.

F. Pasini, S. Gardini, G. L. Marcazzan,
and M. F. Caboni, "Buckwheat $0122-820 \mathrm{X}$
honeys: screening of composition and
properties," Food Chemistry, vol. 141,
pp. 2802-2811, December 2013.
M. Mizuno and M. J. Pikal, "Is the
pre-Tg DSC endotherm observed with
solid state proteins associated with the
protein internaldynamics? Investigation
of bovine serum albumin by solid
state hydrogen/deuterium exchange,"
European Journal of Pharmaceutics
and Biopharmaceutics, vol. 85, pp.
170-176, October 2013.
J. C. Martinez, A. R. Viguera, L. Serrano,
V. V. Filimonov, and P. L. Mateo, "The
DSC data analysis for small, single-
domain proteins. Application to the
SH3 domain," Reactive and Functional
Polymers, vol. 36, pp. 221-225, May
1998.

F. Pasini, S. Gardini, G. L. Marcazzan,
and M. F. Caboni, "Buckwheat $0122-820 \mathrm{X}$
honeys: screening of composition and
properties," Food Chemistry, vol. 141,
pp. 2802-2811, December 2013.
M. Mizuno and M. J. Pikal, "Is the
pre-Tg DSC endotherm observed with
solid state proteins associated with the
protein internaldynamics? Investigation
of bovine serum albumin by solid
state hydrogen/deuterium exchange,"
European Journal of Pharmaceutics
and Biopharmaceutics, vol. 85, pp.
170-176, October 2013.
J. C. Martinez, A. R. Viguera, L. Serrano,
V. V. Filimonov, and P. L. Mateo, "The
DSC data analysis for small, single-
domain proteins. Application to the
SH3 domain," Reactive and Functional
Polymers, vol. 36, pp. 221-225, May
1998.

F. Pasini, S. Gardini, G. L. Marcazzan,
and M. F. Caboni, "Buckwheat $0122-820 \mathrm{X}$
honeys: screening of composition and
properties," Food Chemistry, vol. 141,
pp. 2802-2811, December 2013.
M. Mizuno and M. J. Pikal, "Is the
pre-Tg DSC endotherm observed with
solid state proteins associated with the
protein internaldynamics? Investigation
of bovine serum albumin by solid
state hydrogen/deuterium exchange,"
European Journal of Pharmaceutics
and Biopharmaceutics, vol. 85, pp.
170-176, October 2013.
J. C. Martinez, A. R. Viguera, L. Serrano,
V. V. Filimonov, and P. L. Mateo, "The
DSC data analysis for small, single-
domain proteins. Application to the
SH3 domain," Reactive and Functional
Polymers, vol. 36, pp. 221-225, May
1998.

[29] A. E. Lyubarev and B. I. Kurganov, "Analysis of DSC Data Relating to Proteins Undergoing Irreversible Thermal Denaturation," Journal of Thermal Analysis and Calorimetry, vol. 62, pp. 51-62, October 2000.

[30] G. Bruylants, J. Wouters, y C. Michaux, "Differential scanning calorimetry in life science: thermodynamics, stability, molecular recognition and application in drug design," Curr Med Chem, vol. 12, pp. 2011-2020, 2005.

0122-820X

ISSN 2422-5053

PP: $135-144$ 
analysis of proteins: qualification of DSC," J Pharm Sci, vol. 101, pp. 955964, March 2012.

[33] J. W. Lee, L. C. Thomas, and S. J. Schmidt, "Investigation of the Heating Rate Dependency Associated with the Loss of Crystalline Structure in Sucrose, Glucose, and Fructose Using a Thermal Analysis Approach (Part I)," Journal of Agricultural and Food Chemistry, vol. 59, pp. 684-701, Jan 2011. 